\title{
Real Time Focusing and Directional Light Projection Method for Medical Endoscope Video
}

\author{
Yuxiong Chen, Ronghe Wang, Jian Wang, and Shilong Ma
}

\begin{abstract}
The existing medical endoscope is integrated with a small fixed focus lens in front of small data pipe. After endoscope into disease region, the fixed lens can't transform the focal length according to environment, which leads to surrounding environment can't be seen in the process of endoscopy. This will cause irreversible damage of lesion area. We use the function of first shooting and then focusing and automatic focusing to complete endoscopic process, and transform the environment from fuzzy to high definite by software to adjust focus. It avoids the damage caused by the aggressive of the medical endoscope. At the same time, we design a direction light transmission channel optical fiber to project the direction light for environment that light is not enough, which make the light sufficient and make the endoscopic channel clear. Our medical endoscope shoots and stores video in the endoscopic processing. It also can see clearly everything after completed endoscopic processing, so as to realize multiple diagnoses by one video. The video shot by our endoscope can be used as treatment evaluation materials in the whole treatment process. We use the light video camera focus function in the medical endoscope field firstly. Medical endoscope technical filed is expanded and improved.
\end{abstract}

Index Terms - Light field capture, medical light field camera, micro lens array of light field, projection direction light.

\section{INTRODUCTION}

The limitation of the traditional endoscope is that the video only has one focus. Especially medical endoscope needs to work in a small environment, so it only can use prime lens and cannot use zoom lens. Therefore the whole scene in the focal plane is fuzzy, only a few parts are clear. In the paper, we will use digital endoscope imaging technique different from traditional endoscope. The overall volume of optical field endoscope is small. Because we don't have to choose focus in the shooting processing, so the shooting speed is faster than traditional endoscope. It can use the focus software to focus video after the completion of endoscopy. Through light field technology [1], no matter shooting video is fuzzy or not, as long as the scope is within the endoscopic focal length, the focus can be arbitrary chosen after shooting video. All optical information was recorded in the endoscopic video. The light field endoscope can be used for light field description,

Manuscript received July 18, 2017; revised October 22, 2017.

Yuxiong Chen is with Guangdong Nanfang Vocational College, Guangdong, CO 529000 China (e-mail: 35524677@ qq.com).

Ronghe Wang and Shilong Ma are with State Key Laboratory of Software Development Environment, Beihang University, Beijing, CO 100191 China (e-mail: wangronghe@buaa.edu.cn, slma@nlsde.buaa.edu.cn).

Jian Wang is with Guangdong Nanfang Vocational College, Guangdong, CO 529000 China (e-mail: $23961359 @$ qq.com). synthetic aperture imaging, and multi-view stereo display.

\section{BACKGROUND}

A number of problems in the field of medical endoscope are not well solved for a long time [2].

Due to the precision requirement, medical endoscopes need to integrate $5000 \sim 6000$ cables within $2 \sim 3 \mathrm{~mm}$ thick medical optical fiber. There is only a smaller fixed focal length endoscope lens in front of the cable. When it gets into the patient lesion site, it cannot adjust the focal length according to the changes of surrounding environment [3].

In the current medical endoscope field, the diagnosis needs to repeat for the patient in different hospitals [4], [5]. Every diagnosis means pain and pain.

In the endoscopic processing, video and light field data are not recorded. So the medical endoscope can't refocus on different targets by video, and can't be used as a medical treatment evaluation and accountability system, and can't use optical field micro lens array and related software system automatic projection direction light, and also can't adjust light intensity, and has no automatic focusing system [6]-[8].

This paper researches the field of binding domain of light field camera and medical endoscope. Currently, the light field theory has not yet been applied to medical endoscope field. The technologies of endoscope automatic focusing, first shooting and then focusing and directional light projection are firstly proposed in this paper.

\section{RELATED WORK}

In 1936, Gershun et al. proposed the light field concept [9]. In 1992, Adelson applied light-field theory to computer vision and proposed all-optical field theory (plenoptic theory) [10]. In 1996, Levoy proposed light field rendering theory (light field rendering). In 2005, $\mathrm{Ng}$ invented the first handheld light field camera. In 2006, Levoy developed a light-field microscope. Rendering theoretical is based on Levoy light field [6]. Any information of light intensity and direction in the space, we can use two parallel planes parametric representation, as shown in the following figure,
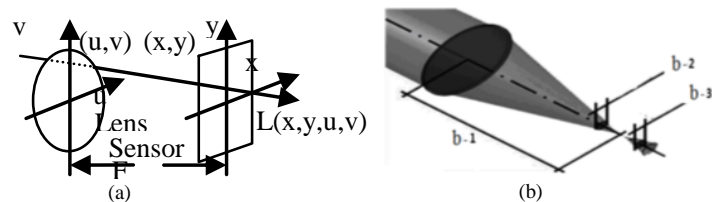

Fig. 1. Light field calculation.

Light and two planes intersect at two points. They form a 
four-dimensional function $L(U, V, x, y)$ [11], [12]. The classical radiation theory shows that the point in image plane is about all radiation weighted integral from the lens [13], as shown in the following,

$$
E_{F}(x, y)=\frac{1}{F^{2}} \iint L_{F}(x, y, u, v) \cos ^{4} \theta d u d v
$$

$L_{F}(x, y, u, v)$ is optical field parameter of distance in the target plane $F$, and is the attenuation factor of optical vignetting effect. Core algorithm includes micro lens array capturing light field, conversion and storage. Light field capture means a ray in the scene can be represented by lens plane and focal plane, and this will capture every ray and will record the scene. If the focus point falls exactly on the focal plane, then it is clear. And this parallel light rays are clear. If the focus point falls inside or outside the plane of focus, then this point is not clear, the light parallel with the light rays are not clear [14]. As shown in the following figure,

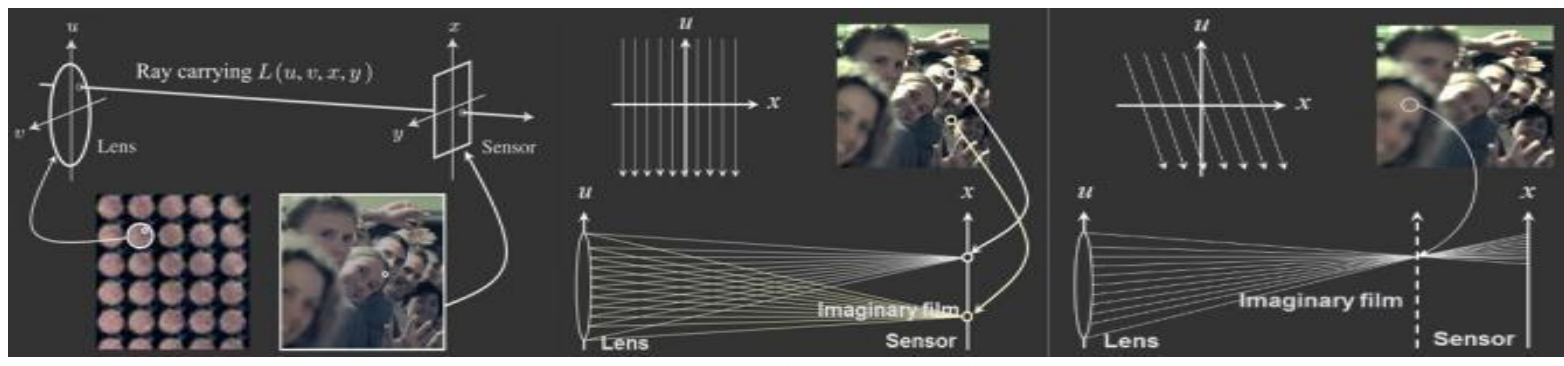

Fig. 2. The process of capturing light field.

Focusing system focuses the same group light to focus plane. Conversion and storage use Fourier transform domain. It is transformed into the frequency domain so as to reduce the storage space. As shown in the following,

$$
g(f(x))=f(g(x))
$$

$g(x)$ means light field transform, $f(x)$ means image transform. We convey light in optic fiber, so as to focus light to specific areas to observe and shadow tiny images onto a photosensitive device. All hazy halos around the focus image become clear, and it keeps the traditional endoscopic large aperture by increasing luminosity. It also reduces patient's pain without sacrificing field depth and image clarity [15].

Smaller aperture is needed for traditional endoscope accessing to large depth of field. In order to balance the signal noise ratio, we increase aperture and decrease depth of field. As shown in the following figure,

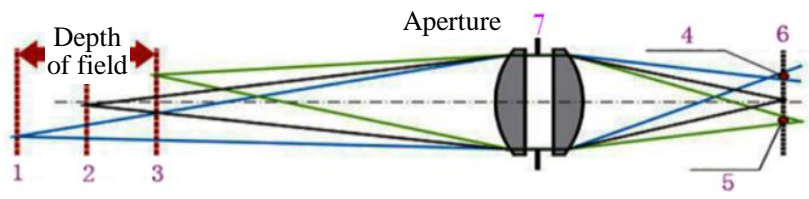

Fig. 3. Depth figure.

In order to balance the signal noise ratio, it is necessary to extend the exposure time, however, the camera shake causes image blur.

\section{LIGHT FIELD MiCRO LENS ARRAY}

We design the optical field medical endoscope composed by the display, CCD chip (1/3 color camera), light field video micro lens array, probe lighting light source, lens rod diameter, built-in power supply, USB power board, a lithium battery and a processor boards, variable pitch control sensor, LCD display screen and a radio circuit board. Among them, the light field video camera is composed by micro lens array and optical field sensor. Light field video camera in each micro lens receives light, before transferring to the CCD chip.
The light scene is recorded by digital, and the digital optical field sensors can collect all light color, intensity and direction into the camera. Micro lens is an 11 million ray resolution camera. That is to say it can capture the 11 million beam of light. Tracking each beam of the light in different distance image, it can take a perfect video, and detect surface defects in non-visible parts and parts that can't be touched by conventional nondestructive testing. The micro lens array is shown below,

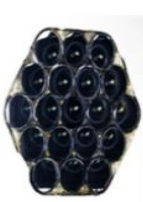

(a)

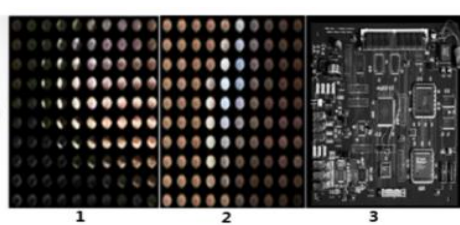

(b)

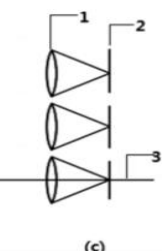

Fig. 4. Light field video camera and light field sensor.

Fig. 4 is the schematic of light field video camera and light field sensor. Fig. 4(a) shows micro lens array hexagonal arrangement. Fig. 4(b) shows micro lens array arrangement in the light field sensor. Fig. 4(b)-1 shows a lens exposure. Fig. 4(b)-2 shows another exposure. Fig. 4(b)-3 shows the connection diagram of micro lens array in the light field sensor. Fig. 4(c) shows sketch map of main lens and micro lens in the light field sensor links. Fig. 4(c)-1 shows micro lens. Fig. 4(c)-2 shows micro lens optical field sensors link. Fig. 4(c)-3 shows micro optical axis of the lens.

An exposure and record of micro-lens is shown below,

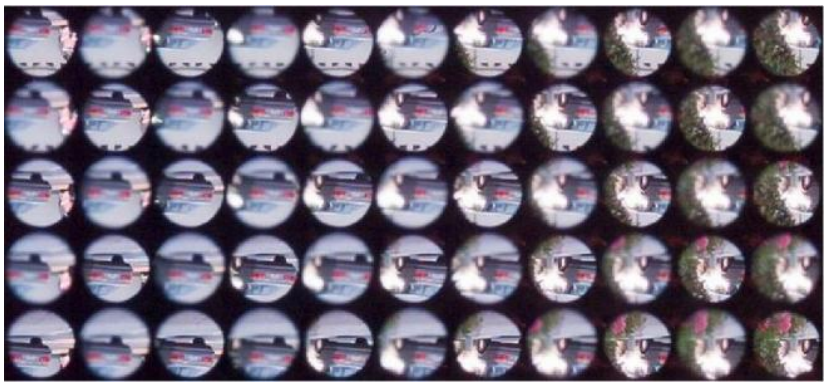

Fig. 5. An exposure and record of micro-lens

The light field endoscope can capture any transmission 
direction light within the field of view. Its characteristic is mainly the light field video camera real-time arbitrary perspective focusing function. The external object image is automatically focused through a series of optical system and is focused clearly on the screen. The endoscope can automatically change focusing according to environment changes and probe depth, and can show a clear picture, vivid color, high resolution. There is no way to change the view angle to observe the spatial structure of small objects, but the endoscope shooting video can reposition observation from many view point. Endoscopic depth has been measured inside the object and movement is no longer controlled by software on the screen to see small objects from different perspective. The lens design is shown below,

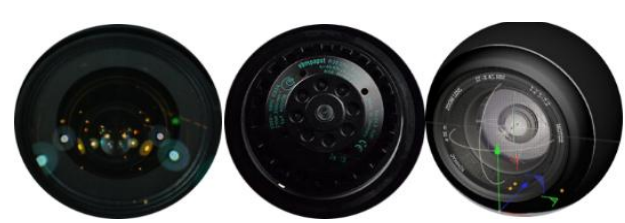

Fig. 6. Endoscopic lens design.

Light field video camera micro lens array mainly uses the function of first shooting and then focusing to complete endoscopy. It solves the problem of medical probe depth detection. It solves the problem that the medical probe cannot move and rotate [4] inside the diseased organ. It changes view angle, clearly shows every scene corner, and then completes endoscopy. If the endoscope has not zoom lens, it can only use fixed focus lens to focus and see straight ahead. The micro-lens spotlight schematic is shown below,

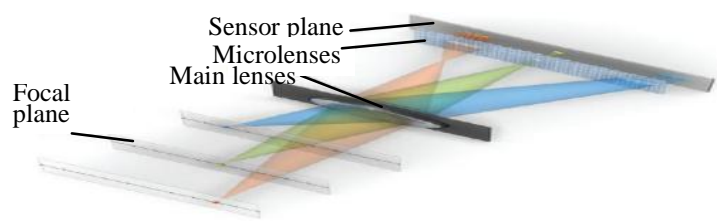

Fig. 7. The micro-lens spotlight schematic.

When the endoscope is unable to move or it is inconvenient to move because of deep lesions, the movement can cause adverse reactions of patients or serious damage to organs. In the case of unknown disease severity, this huge medical endoscope equipment will injure intensive patient organ, and this is irreversible. In this paper, the endoscope in patient depth diseased area is placed to a suitable location to focus the scene seen by light field camera software system. The optical fiber with a micro lens array control software system shoots 30 times per second, and probably spreads about 30 frames. It's the processing of real-time video stream.

\section{Directional OpticAl FiBER}

Optical fiber is used to project directional light, such as internal lighting, and it is also used to output images. It is shown in the following figure,

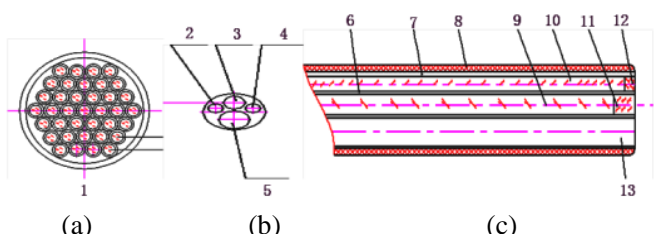

Fig. 8. Directional light transmission optical fiber structure.
Fig. 8 is medical optical fiber structure diagram. Fig. 8 (a) is hexagonal arrangement of fiber. Fig. 8(b)-2 is mechanical pathways in fiber cross-section. Fig. 8(b)-3 is fiber cross-section lighting window. Fig. 8(b)-4 is window lens fiber cross-section. Fig. 8(b)-5 is the cross-section of fiber lighting window. Fig. 8(c)-6 is fiber CCD video line. Fig. $8(\mathrm{c})-7$ is inner soft casing. Fig. $8(\mathrm{c})-8$ is outer protective sleeve. Fig. 8(c)-9 is optical fiber transmission. Fig. 8(c)-10 is fiber optic lighting. Fig. 8(c)-11 is an objective lens. Fig. 8 (c)-12 is lighting lenses. Fig. 8(c)-13 is mechanical channel.

The basic focused plenoptic rendering algorithm is shown in the following figure,

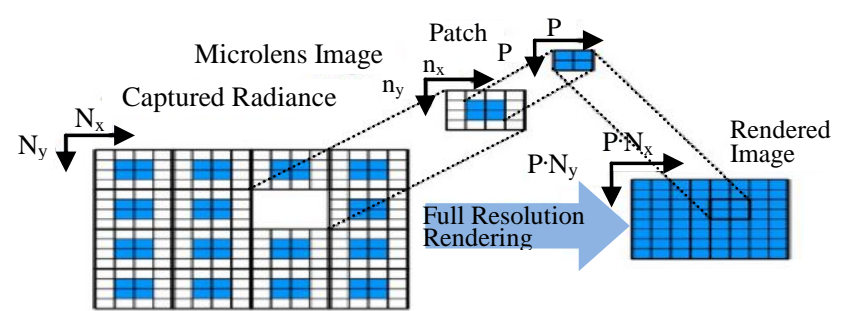

Fig. 9. Basic focused plenoptic rendering algorithm creates a final rendered image from $P^{*} P$ patches of each $n_{x}^{*} n_{y}$ microimage. With $N_{X} * N_{Y}$ microimages in the captured radiance, the final rendered image is $P^{*} N_{x}^{*} P^{*} N_{y}$

When the endoscope is deeply into the disease parts of the human body, we can adjust the focus and see disease dirty parts clearly by the terminal computer software system. It can overcome the problem that the traditional endoscope can only clearly see drawback of the surrounding organs and lesions in front of the endoscope, can reduce the lesion injury and pain. It is fixed-point observation, one by one to into, steadily further. The directional lighting system is also used for lighting, as shown in the following figure,

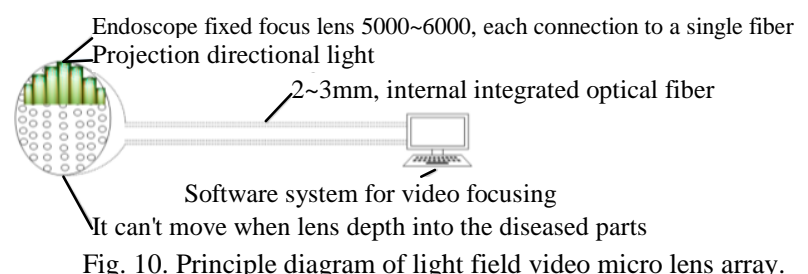

Our main contribution is to solve the endoscopic video streaming high-resolution imaging, continuous editing and endoscopic procedure to see every corner of the scene. The video stream is captured directly at arbitrary angle of the optical field and can be focused at arbitrary angle. As shown in the following figure,
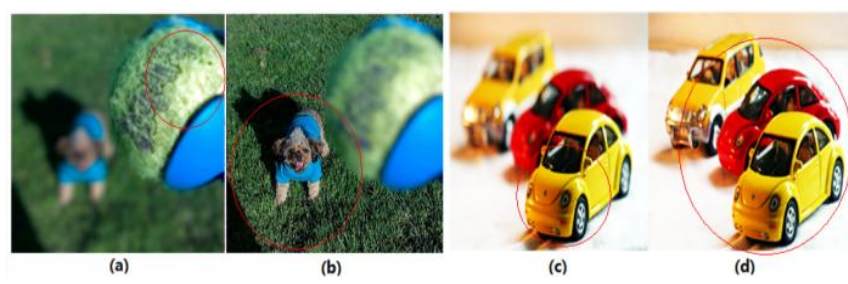

Fig. 11. Light field focusing effect chart

Fig. 11 shows focus on two frame of the video. Fig. 11(a) and Fig. 11(b) are two focusing on one frame taken. Fig. 11(c) and Fig. 11(d) are another frame of another video. The circle is focus point. 


\section{CONNECTION MODE}

The design structure of our medical endoscope is shown in the following figure,

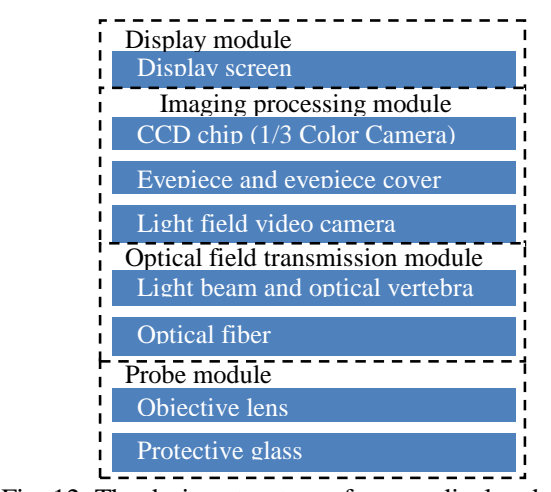

Fig. 12. The design structure of our medical endoscope.

In the figure, display module embeds a display at the top of the medical endoscope. CCD chip ( $1 / 3$ color camera), eyepiece cover and eyepiece, and light field video camera micro lens array compose light field imaging processing module. Light beam and optical vertebra, optical fiber compose optical field transmission module. Objective lens and protective glass compose probe module. Each module is connected in turn. Optical fiber is placed on the searchlight illumination handheld hose. Endoscopic probe installs a lighting light source at one end of the hose. When the probe is deeply into internal of the patient body, scene through the optical fiber afferents light inside the endoscope and the endoscope through its own focusing function shows a clear image. CCD chip ( $1 / 3$ color camera) is for storage and display. The imaging relies mainly on endoscope of video camera sensor, micro lens. Entire column of light field video camera likes a miniature camera. Scene is focused processing and displayed on a TV monitor in the display. The video refocus focusing after video shoot. CCD chip (1/3 color camera), eyepiece cover and eyepiece are connected to the light field video camera. Image is transmitted by optical fiber and endoscopic probe of the lens. Light is automatically focused processing through the optical fiber and light endoscope channels, and the image is clearly displayed on the display. As shown in the following figure,

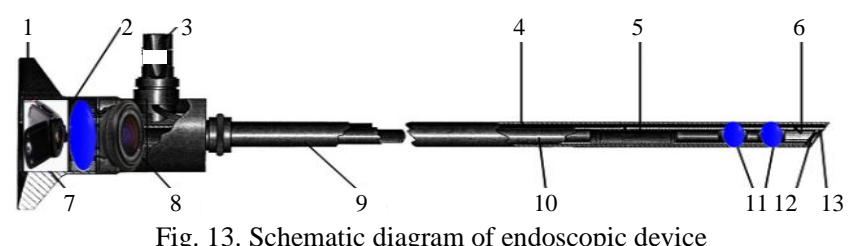

Fig. 13 is the design of medical light endoscope stereo view. Fig. 13-1 is for display. Fig. 13-2 as ocular. Fig. 13-3 is the optical axis and the light cone. Fig. 13-4 is illumination fiber. Fig. 13-5 is lens. Fig. 13-6 is 30 degrees angle prism. Fig. 13-7 is CCD chip (1/3) camera. Fig. 13-8 is light field video camera micro lens array. Fig. 13-9 is mirror tube. Fig. 13-10 is endoscope. Fig. 13-11 is lens. Fig. 13-12 is negative lens. Fig. 13-13 is protection. The components are connected in turn according to the graph.

The larger the aperture (smaller F-measure), the more light, the depth of field is smaller, and the main is more highlight. The endoscopic probe shoots the video with axial and longitudinal resolution. Our method has greater flexibility and greater depth of field, does not need to focus accurately. Different from Levoy light field camera, the light field video camera micro lens array uses only one layer of the lens inside the micro lens, one fiber connected with a micro lens array, about 5000 to 6000 micro lenses. When the inner endoscopic probe depth has been detected in the interior of patient, scene is transmitted to the light field video camera micro lens array through the optical fiber, and then the light field video camera shows real-time clear video image through its autofocusing function. High quality imaging mainly depends on light field video camera micro lens array and image sensor. Light field video camera likes a miniature camera. Scene is processed by the image processor and displayed on the monitor of the endoscope. As shown in the following figure,

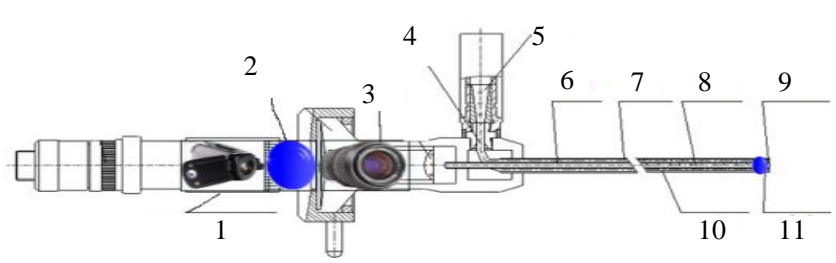

Fig. 14. Internal configuration of the endoscope

Fig. 14 is endoscopic structure. Fig. 14-1 is CCD chip (1/3) camera. Fig. 14-2 as ocular. Fig. 14-3 is light field video camera. Fig. 14-4 is optical axis. Fig. 14-5 is light cone. Fig. 14-6 is casing. Fig. 14-7 is the outer tube. Fig. 14-8 is passing like fiber. Fig. 14-9 is protective glass. Fig. 14-10 is fiber optic lighting. Fig. 14-11 is an objective lens.

Images are focused to the light field video camera optical sensor in the endoscopic process. It will record the sum of all the light in the photo on the spot. Each small lens array of the micro lens array receives rays and transfers them to the CCD chip. Through light data conversion, according to the need, the focus is chosen. The last video imaging effect can also be done on a computer. After a lapse of several years user can also use other methods to adjust focus, and can reselect perspective to observe endoscopic materials, which can be as effective evidence of surgery and treatment.

\section{CONTRIBUTION POINT OF THIS PAPER}

Through the endoscopic probe, objective lens, fiber, eyepiece, light field video camera, CCD camera, transmission channel and light field video camera, light field is focused and shown on the screen to make the picture clear. Using light inside the endoscope, the optical field endoscope can focus and zoom. Image is clear, realistic, and can be magnified. The mucosal and fine structure can be observed. Under low light and relative high speed mobile, the peep mirror can still focus accurately and shoot clear video to capture a large number of light data and select focus. Focus can be chosen in free after shoot, because all optical information of endoscope taking pictures in the focal length range is recorded. It can change the angle of view for watching the video. In this paper, the endoscope equipment fully captures light field to make imaging more clear and precision. Even the video was shot a 
number of years, the moment you can get what you want from different perspectives of ideal video. Its effect as shown in the following figure,
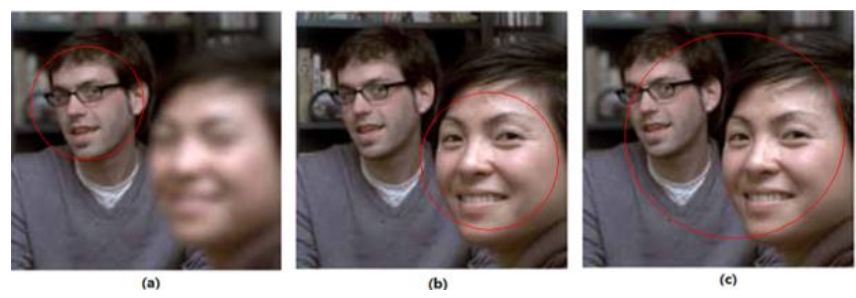

Fig. 15. Focus effect diagram.

Fig. 15 shows two different perspective focuses after the shooting of the video. Fig. 15(a) shows an original image flame in the original video. Fig. 15(b) shows a focus. Fig. 15(c) shows another focus. The circle is focus point.

\section{CONCLUSION}

This paper proposed a first shooting and then focusing technique in the field of industrial endoscope and medical endoscope, which expands and improves the two endoscope technology fields. We also gave the light field video camera and its practical application example. Using light field video camera software, we change the view point to complete a clear endoscopy. We truly achieve the automatic focusing, real-time focusing and high-definition display of the endoscopic probe, so that users can real-time observe in detail from different perspectives in the wake of the shooting.

(1) We change the medical fiber $(2-3 \mathrm{~mm})$ front fixed focus lens to fit light field calculation zoom micro lens array. The focal length of the lens can be adjusted automatically by computer terminal software system, so as to eliminate the video fuzzy problem because the endoscope can't zoom lens in patient lesion. It avoids irreversible injury of patient organ.

(2) We increase projection directional light at the front of the lens to ensure that the endoscope environment is in the fiber light sufficient conditions. It's used to automatically calculate according to dark scene. If somewhere is lightless, we will cast a bright light on key areas.

(3) After completing the whole endoscopic video, we use software system to focus lesion. We can focus the target again and clearly observe any target in the scene.

(4) Because we record whole optical field in the processing, we can refocus arbitrary goal of the scene. Because subsequence diagnosis is repeat focus in the original video, we achieve an endoscope to complete multiple diagnoses, and we reduce the patient injury times to the only once. The endoscopic video can be used as treatment evaluation system and relevant evidence accountability system.

\section{ACKNOWLEDGMENT}

We sincerely thank each one of the reviewer and editors' work to the paper.

\section{REFERENCES}

[1] X. Zhang, L. Dong, Q. and H. Zhang, "Method of three-dimensional localization and tracking of magnetic field in endoscope," Chinese Journal of Biomedical Engineering, May 2012.
[2] K. Mitra and A. Veeraraghavan. "Light field denoising, light field superresolution and stereo camera based refocussing using a GMM light field patch prior," in Proc. IEEE CVPR Workshops, 2012, pp. 22-28.

[3] S. Wanner and B. Goldlucke, "Globally consistent depth labeling of $4 \mathrm{~d}$ light fields," in Proc. IEEE CVPR Workshops, 2012, pp. 41-48.

[4] S. Wanner and B. Goldluecke, "Spatial and angular variational super-resolution of $4 \mathrm{~d}$ light fields," in Proc. IEEE ECCV, 2012, pp. 608-621.

[5] Ng R, Levoy M, Bredif M, "Light field photography with a hand-held plenoptie camera," Teeh Rep CSTR: Stanford Computer Science Tech Report CSTR, 2005.

[6] M. Levoy, R. Ng, and A. Adams, "Light field microscopy," Acm Transactions on Graphics, vol. 25, no. 3, pp. 924-934, 2006.

[7] Y. Yuan, Y. Zhou, H. and H. Hu, "Analysis of the registration error of the micro lens array and detector in the light field camera," Photon Journal, vol. 39, no. 1, pp. 123-126, 2010.

[8] N. Li, J. Ye, and Y. Ji, "Saliency detection on light field," in Proc. IEEE CVPR Workshops, 2014, pp. 2806-2813.

[9] H. Lin, C. Chen, and S. B. Kang, "Depth Recovery from light field using focal stack symmetry," in Proc. IEEE International Conference on Computer Vision, 2015, pp. 3451-3459.

[10] O. Johannsen, A. Sulc, and B. Goldluecke, "On linear structure from motion for light field cameras," in Proc. IEEE International Conference on Computer Vision, 2015, pp. 720-728.

[11] Z. Yu, X. Guo, and H. Ling, "Line assisted light field triangulation and stereo matching," in Proc. EEE International Conference on Computer Vision, 2013, pp. 2792-2799.

[12] S. Tambe, A. Veeraraghavan, and A. Agrawal, "Towards motion aware light field video for dynamic scenes," pp. 1009-1016, 2013.

[13] C. Chen, H. Lin, and Z. Yu, "Light field stereo matching using bilateral statistics of surface cameras," in Proc. IEEE Conference on Computer Vision and Pattern Recognition, 2014, pp. 1518-1525.

[14] K. Maeno, H. Nagahara, and A. Shimada, "Light field distortion feature for transparent object recognition," in Proc. IEEE CVPR Workshops, 2013, pp. 2786-2793.

[15] G. Wetzstein, D. Roodnick, and W. Heidrich, "Refractive shape from light field distortion," in Proc. IEEE International Conference on Computer Vision, Barcelona, Spain, November, pp. 1180-1186, 2011.

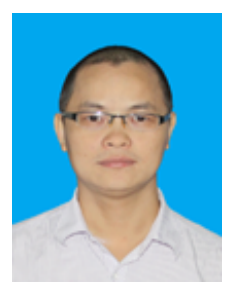

Yuxiong Chen is a master, lecturer, and outstanding young teacher of Guangdong Province. He is working in Guangdong Nanfang Vocational College. He is one of team leaders of Software Technology Specialty. He is a responsible person for College of software technology specialty construction. His research interests include image processing, computer software and theory.

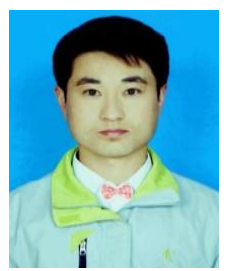

Ronghe Wang received his MS degrees from Beihang University, Beijing, China, in 2012. He is working toward his Ph.D. degree in computer science and technology at the School of Computer Science and engineering in Beihang University currently, Beijing, China. His research interests include image processing, computer vision, virtual reality, medical visualization, computer software, theory and so on.

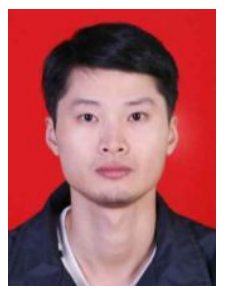

Jian Wang was born in 1980 . He is now a lecturer and is working in Guangdong Nanfang Vocational College. $\mathrm{He}$ is deputy director of teaching and research section of computer application technology. His research interests include image processing, computer software and theory.

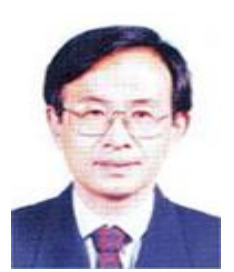

Shilong Ma was born in 1953. He is Professor and $\mathrm{Ph} . D$. supervisor of School of Computer Science and Engineering in Beihang University. He is working in the China State Key Laboratory of Software Development Environment. His research interests are in the field of computation models in network, logic reasoning and behaviors in network computing, theory of automatic test, etc. 